EUROPEAN JOURNAL OF PURE AND APPLIED MATHEMATICS

Vol. 14, No. 4, 2021, 1517-1529

ISSN 1307-5543 - ejpam.com

Published by New York Business Global

\title{
The Bivariate Extended Poisson Distribution of type 1
}

\author{
Rufin Bidounga ${ }^{1,3, *}$, Michel Koukouatikissa Diafouka ${ }^{1,3}$, Réolie Foxie Mizélé \\ Kitoti $^{2,3}$, Dominique Mizère ${ }^{2,3}$ \\ ${ }^{1}$ Exact Science Department, Normal School Higher, Marien Ngouabi University, \\ Brazzaville, Congo \\ ${ }^{2}$ Department of Mathematics, Faculty of Science and Technology, \\ Marien Ngouabi University, Brazzaville, Congo \\ ${ }^{3}$ Laboratory of statistics and analysis of data (Labsad), Faculty of Science and Technology, \\ Marien Ngouabi University, Brazzaville, Congo
}

\begin{abstract}
In this paper, we will construct the bivariate extended Poisson distribution which generalizes the univariate extended Poisson distribution. This law will be obtained by the method of the product of its marginal laws by a factor. This method was demonstrated in [7]. Thus we call the bivariate extended Poisson distribution of type 1 the bivariate extended Poisson distribution obtained by the method of the product of its marginal distributions by a factor. We will show that this distribution belongs to the family of bivariate Poisson distributions and and will highlight the conditions relating to the independence of the marginal variables. A simulation study was realised.
\end{abstract}

2020 Mathematics Subject Classifications: 62E15, 62H10, 60E05

Key Words and Phrases: Extended Poisson distribution, bivariate Poisson distribution, estimation and statistical testing

\section{Introduction}

Several authors have studied bivariate Poisson laws, in particular, Berkhout and Plug[2] and Lakshminarayna et al.[7]. Then, [3] has highlighted the weighted bivariate Poisson law having as a basic law, the bivariate Poisson law according to Berkhout and Plug[2]; a law that allows to generate all the bivariate Poisson laws. The bivariate Poisson distribution according to Berkhout and Plug[2] is rightly considered the standard distribution in $\mathbb{N}^{2}$ as is the Poisson distribution in $\mathbb{N}$. In this paper, we will construct the bivariate extended Poisson distribution which generalizes the univariate extended Poisson distribution. This law is obtained by the method of the product of its marginal laws by a factor. This method was demonstrated in [7], thus we call the bivariate extended Poisson distribution of type

${ }^{*}$ Corresponding author.

DOI: https://doi.org/10.29020/nybg.ejpam.v14i4.4151

Email addresses: rufbid@yahoo.fr(R. Bidounga), michel.koukouatikissa@umng.cg (M. Koukouatikissa Diafouka), foxie_reolie2000@yahoo.fr (R. F. Mizélé Kitoti), domizere@gmail.com (D. Mizère) 
1 the bivariate extended Poisson distribution obtained by the method of the product of its marginal distributions by a factor. We have shown that this distribution belongs to the family of bivariate Poisson distributions and have highlighted the conditions relating to the independence of the marginal variables. A simulation study was realised.

\section{A review of laws}

\subsection{The Univariate Extended Poisson distribution}

Definition 1. The laws following probability mass function

$$
P(Y=y)=\left\{\begin{array}{ll}
\frac{1-e^{-\theta}}{\beta}, & y=0, \\
\left(\frac{\theta^{y}}{y !} e^{-\theta}\right) \beta^{-1}\left(\frac{\beta}{\theta} y-1\right), & y=1,2, \ldots
\end{array} \quad \forall \theta>0, \text { and } \beta \geq \theta,\right.
$$

is renamed as the univariate extended Poisson distribution (see [5]).

It can be written in the form [8]

$P(Y=y)=\frac{\theta^{y}}{y !} e^{-\theta}\left(\frac{\beta}{\theta} y-1\right) \beta^{-1}\left\{\frac{1-e^{-\theta}}{\left(\frac{\beta}{\theta} y-1\right) e^{-\theta}}\right\}^{\delta_{0}(y)} \quad, \quad y \in \mathbb{N}, \forall \theta>0$ and $\beta \geq \theta$,

where $\delta_{0}$ is the Dirac function in $0 . \theta$ is the canonic parameter. This distribution has the following characteristics

$$
\begin{aligned}
E_{\theta}(Y) & =1+\frac{\beta-1}{\beta} \theta, \\
\operatorname{var}(Y) & =\frac{\beta-1}{\beta^{2}} \theta^{2}+\frac{\beta+1}{\beta} \theta .
\end{aligned}
$$

\section{Under- or over-dispersed distribution}

The following facts are immediate.

Proposition 1. The Fisher dispersion index of the variable $Y$ which follows the extended Poisson distribution of parameters $(\theta, \beta)$ noted $I(Y)$ is such that

- $I(Y)>1$ if $\frac{\beta}{1+\sqrt{\beta}}<\theta \leq \beta$, i.e. the extended Poisson distribution is overdispersed 
- $I(Y)<1$ if $0<\theta<\frac{\beta}{1+\sqrt{\beta}}$, i.e. the extended Poisson distribution is underdispersed

- $I(Y)=1$ if $\theta=\frac{\beta}{1+\sqrt{\beta}}$, i.e. the extended Poisson distribution is equiderdispersed.

Proof. Indeed,

$$
\begin{aligned}
\operatorname{var}(Y)-E(Y) & =\frac{\beta-1}{\beta^{2}} \theta^{2}+\frac{\beta+1}{\beta} \theta-1-\frac{\beta-1}{\beta} \theta, \\
& =\frac{\theta^{2} \beta-(\beta-\theta)^{2}}{\beta^{2}}, \\
& =\frac{(\theta \sqrt{\beta}+\beta-\theta)[\theta(1+\sqrt{\beta})-\beta]}{\beta^{2}} .
\end{aligned}
$$

Since $\beta \geq \theta>0$ then the sign of $\operatorname{var}(Y)-E(Y)$ depends only on $\theta(1+\sqrt{\beta})-\beta$. Then $I(Y)$ is greater, smaller or equal to 1 depending on whether $\operatorname{var}(Y)-E(Y)$ is positive, negative or null respectively. We are assured of the answer

Let us recall the result of [5].

Proposition 2. The moments generating function of the extended Poisson distribution is equal to

$$
M_{Y}(t)=\frac{1-\left(1-\beta e^{t}\right) e^{\theta\left(e^{t}-1\right)}}{\beta}, \quad t \in[-1,1] .
$$

Now, we have the following result.

\section{Proposition 3.}

$$
\begin{aligned}
E_{\theta}\left[e^{-Y}\right] & =\frac{1-\left(1-\beta e^{-1}\right) e^{\theta\left(e^{-1}-1\right)}}{\beta}, \\
E_{\theta}\left[\left(e^{-Y}\right)^{2}\right] & =\frac{1-\left(1-\beta e^{-2}\right) e^{\theta\left(e^{-2}-1\right)}}{\beta}, \\
E_{\theta}\left[Y e^{-Y}\right] & =\frac{e^{-1} e^{\theta\left(e^{-1}-1\right)}\left[\beta-\theta\left(1-\beta e^{-1}\right)\right]}{\beta} .
\end{aligned}
$$

Proof. Indeed, expression (6) is obvious because it is equal to $M_{Y}(-1)$. Ditto for expression (7) which is equal to $M_{Y}(-2)$. And for expression (8), we have $E_{\theta}\left(Y e^{t Y}\right)=$ $\frac{d}{d t} M_{Y}(t)$. Since $\frac{d}{d t} M_{Y}(t)=\frac{e^{t} e^{\theta\left(e^{t}-1\right)}\left[\beta-\theta\left(1-\beta e^{t}\right)\right]}{\beta}$, by setting $\mathrm{t}=-1$, we are assured of the answer. 


\subsection{The Bivariate Poisson distribution according to Berkhout and Plug[2]}

Definition 2. Let $Y_{j}(j=1,2)$ be a random variable that follows the Poisson distribution of parameter $\theta_{j}(j=1,2)$. The vector $\left(Y_{1}, Y_{2}\right)$ follows the bivariate Poisson distribution according to Berkhout and Plug [2] if its mass function $f_{B P}$ is equal to

$$
f_{B P}\left(y_{1}, y_{2} ; \theta_{1}, \theta_{2}\right)=\left(\frac{\theta_{1}^{y_{1}}}{y_{1} !} e^{-\theta_{1}}\right)\left(\frac{\theta_{2}^{y_{2}}}{y_{2} !} e^{-\theta_{2}}\right), \quad\left(y_{1}, y_{2}\right) \in \mathbb{N}^{2},\left(\theta_{1}, \theta_{2}\right) \in \mathbb{R}_{+}^{* 2},
$$

under conditions

$$
\begin{aligned}
& \ln \theta_{1}=x^{\prime} \rho_{1}, \\
& \ln \theta_{2}=x^{\prime} \rho_{2}+\eta y_{1},
\end{aligned}
$$

where $\rho_{1}, \rho_{2}$ and $\eta$ are parameters, $x=\left(x_{1}, \ldots, x_{p}\right)$ is a vector of deterministic variables or factors.

The generalized model (10) has the response variable $Y_{1}$ and the model (11) the variable $Y_{2}$. The expression (10) induces that $P\left(Y_{1}=y_{1} ; \theta_{1}\right)=\frac{\theta_{1}^{y_{1}}}{y_{1} !} e^{-\theta_{1}}$ is a marginal law while the model (11) induces that

$$
\begin{aligned}
P\left(Y_{2}=y_{2} ; \theta_{2}\right) & =P\left(Y_{2}=y_{2} / Y_{1}=y_{1}\right), \\
& =\frac{\theta_{2}^{y_{2}}}{y_{2} !} e^{-\theta_{2}}, \\
& =\frac{e^{y_{2}\left(x^{\prime} \rho_{2}+\eta y_{1}\right)}}{y_{2} !} e^{-\left(x^{\prime} \rho_{2}+\eta y_{1}\right),}
\end{aligned}
$$

is a conditional law.

When $\eta=0$ then the conditional probability $P\left(Y_{2}=y_{2} / Y_{1}=y_{1}\right)$ is not depend of observation $y_{1}$ and the variables $Y_{1}$ and $Y_{2}$ are independent.

The bivariate Poisson distribution according to Berkhout and Plug[2] has the characteristics (see [1]).

$$
\begin{aligned}
& E_{\theta_{1}}\left(Y_{1}\right)=\operatorname{var}\left(Y_{1}\right)=\theta_{1}, \\
& E_{\theta_{2}}\left(Y_{2}\right)=e^{x^{\prime} \rho_{2}+a_{2}+\theta_{1}\left(e^{\eta}-1\right),}
\end{aligned}
$$




$$
\begin{aligned}
\operatorname{var}\left(Y_{2}\right) & =E_{\theta_{2}}\left(Y_{2}\right)+\left[E_{\theta_{2}}\left(Y_{2}\right)\right]^{2}\left(e^{\theta_{1}\left(e^{\eta}-1\right)}-1\right), \\
\operatorname{cov}\left(Y_{1}, Y_{2}\right) & =\theta_{1} E_{\theta_{2}}\left(Y_{2}\right)\left(e^{\eta}-1\right) .
\end{aligned}
$$

Expression (14) shows that the variable $Y_{2}$ is overdispersed. And the covariance is negative, null or positive depending on whether the parameter $\eta$ is negative, null or positive.

\subsection{The Bivariate Poisson distribution according to Lakshminarayna et al. [7]}

In [7], the authors defined the bivariate Poisson law as the product of its marginal laws by a multiplier factor.

Definition 3. Let $Y_{1}$ and $Y_{2}$ be two Poisson random variables with respective parameters $\theta_{1}$ and $\theta_{2}$. The bivariate distribution of the couple $\left(Y_{1}, Y_{2}\right)$, denoted $f_{L P S}$, has the mass function

$$
f_{L P S}\left(y_{1}, y_{2} ; \theta_{1}, \theta_{2}, \alpha\right)=\left(\frac{\theta_{1}^{y_{1}}}{y_{1} !} e^{-\theta_{1}}\right)\left(\frac{\theta_{2}^{y_{2}}}{y_{2} !} e^{-\theta_{2}}\right)\left[1+\alpha\left(e^{-y_{1}}-e^{-d \theta_{1}}\right)\left(e^{-y_{2}}-e^{d \theta_{2}}\right)\right],
$$

with $e^{-d \theta_{i}}=E_{\theta_{i}}\left(e^{-Y_{i}}\right), \quad y_{i} \in \mathbb{N}, \quad \theta_{i} \in \mathbb{R}_{+}^{*}(i=1,2), \quad \alpha \in \mathbb{R}_{+}^{*}$ and $d=1-e^{-1}$.

This distribution has the following characteristics

$$
\begin{aligned}
E_{\theta_{1}}\left(Y_{1}\right) & =\operatorname{var}\left(Y_{1}\right)=\theta_{1}, \\
\operatorname{cov}\left(Y_{1}, Y_{2}\right) & =\theta_{1} \theta_{2} d^{2} e^{-d\left(\theta_{1}+\theta_{2}\right) .}
\end{aligned}
$$

In [4], the authors showed that the bivariate Poisson distribution according to Lakshminarayna et al.[7] is a distribution of the bivariate Poisson family and that it converges to the bivariate Poisson distribution according to Berkhout and Plug[2].

\section{The Bivariate Extended Poisson distribution of type 1}

Based on the work [7], we define the bivariate extended Poisson of type 1 distribution as follows.

Definition 4. Let us consider $Y_{1}$ and $Y_{2}$ two univariate extended Poisson variables with respective parameters $\left(\theta_{1}, \beta_{1}\right)$ and $\left(\theta_{2}, \beta_{2}\right)$. The bivariate Poisson distribution of the pair $\left(Y_{1}, Y_{2}\right)$, denoted $f_{B E P, 1}$, has the mass function (see [7])

$f_{B E P, 1}\left(y_{1}, y_{2} ; \theta_{1}, \theta_{2}, \beta_{1}, \beta_{2}, \alpha\right)=\left(\prod_{j=1}^{2}\left[\left(\frac{\theta_{j}^{y_{j}}}{y_{j} !} e^{-\theta_{j}}\right)\left(\frac{\beta_{j}}{\theta_{j}} y_{j}-1\right) \beta_{j}^{-1}\left\{\frac{1-e^{-\theta_{j}}}{\left(\frac{\beta_{j}}{\theta_{j}} y_{j}-1\right) e^{-\theta_{j}}}\right\}^{\delta_{0}\left(y_{j}\right)}\right]\right) \times$ 


$$
g\left(y_{1}, y_{2} ; \theta_{1}, \theta_{2}, \alpha\right)
$$

where $g\left(y_{1}, y_{2} ; \theta_{1}, \theta_{2}, \alpha\right)=\left[1+\alpha\left(e^{-y_{1}}-c_{1}\right)\left(e^{-y_{2}}-c_{2}\right)\right]$, with $c_{j}=E_{\theta_{j}}\left(e^{-Y_{j}}\right), y_{j} \in \mathbb{N}$, $\theta_{j} \in \mathbb{R}_{+}^{*}, \beta_{j} \geq \theta_{j}(j=1,2)$ and $\alpha \in \mathbb{R}$.

The initials "BEP,1" stand for Bivariate Extended Poisson of type 1. We have the following result.

\section{Proposition 4.}

(i) The marginal laws of $Y_{1}$ and $Y_{2}$ are extended Poisson laws of respective parameters $\left(\theta_{1}, \beta_{1}\right)$ and $\left(\theta_{2}, \beta_{2}\right)$.

(ii)

$$
\operatorname{cov}\left(Y_{1}, Y_{2}\right)=\alpha \operatorname{cov}\left(Y_{1}, e^{-Y_{1}}\right) \operatorname{cov}\left(Y_{2}, e^{-Y_{2}}\right)
$$

Let be $P\left(Y_{j}=y_{j}\right)=\left(\frac{\beta_{j}}{\theta_{j}} y_{j}-1\right) \beta^{-1}\left[\frac{1-e^{-\theta_{j}}}{\left(\frac{\beta_{j}}{\theta} y_{j}-1\right) e^{-\theta_{j}}}\right]^{\delta_{0}\left(y_{j}\right)} \forall \theta_{j}>0, \beta_{j} \geq \theta_{j}$, $j=1,2$, the marginal law of variable $Y_{j} j=1,2$. It follows the result.

\section{Corollary 1.}

$$
f_{B E P, 1}\left(y_{1}, y_{2} ; \theta_{1}, \theta_{2}, \beta_{1}, \beta_{2}, \alpha\right)=P\left(Y_{1}=y_{1}\right) P\left(Y_{2}=y_{2}\right)\left[1+\alpha\left(e^{-y_{1}}-c_{1}\right)\left(e^{-y_{2}}-c_{2}\right)\right],
$$

$c_{j}=E_{\theta_{j}}\left(e^{-Y_{j}}\right), y_{j} \in \mathbb{N}, \theta_{j} \in \mathbb{R}_{+}^{*}, \beta_{j} \geq \theta_{j}(j=1,2)$ and $\alpha \in \mathbb{R}$.

This result confirms that the definition 4 is rigorously correct.

Corollary 2. When $\alpha=0$, the variables $Y_{1}$ and $Y_{2}$ are independent.

Proof. [Proof of the proposition 5]

(i)

$$
\begin{aligned}
P\left(Y_{1}=y_{1}\right)= & \sum_{y_{2}} f_{B E P, 1}\left(y_{1}, y_{2} ; \theta_{1}, \theta_{2}, \beta_{1}, \beta_{2}, \alpha\right) \\
= & \left(\frac{\theta_{1}^{y_{1}}}{y_{1} !} e^{-\theta_{1}}\right)\left(\frac{\beta_{1}}{\theta} y_{1}-1\right) \beta_{1}^{-1}\left\{\frac{1-e^{-\theta_{1}}}{\left(\frac{\beta_{1}}{\theta_{1}} y_{1}-1\right) e^{-\theta_{1}}}\right\}^{\delta_{0}\left(y_{1}\right)} \times \\
& \sum_{y_{2}}\left(\frac{\theta_{2}^{y_{2}}}{y_{2} !} e^{-\theta_{2}}\right)\left(\frac{\beta_{2}}{\theta} y_{2}-1\right) \beta_{2}^{-1}\left\{\frac{1-e^{-\theta_{2}}}{\left(\frac{\beta_{2}}{\theta_{2}} y_{2}-1\right) e^{-\theta_{2}}}\right\}^{\delta_{0}\left(y_{2}\right)}+
\end{aligned}
$$




$$
\begin{aligned}
& \alpha\left(e^{-y_{1}}-c_{1}\right) \sum_{y_{2}}\left(\frac{\theta_{2}^{y_{2}}}{y_{2} !} e^{-\theta_{2}}\right)\left(\frac{\beta_{2}}{\theta} y_{2}-1\right) \beta_{2}^{-1}\left\{\frac{1-e^{-\theta_{2}}}{\left(\frac{\beta_{2}}{\theta_{2}} y_{2}-1\right) e^{-\theta_{2}}}\right\}^{\delta_{0}\left(y_{2}\right)} \times \\
& \left(e^{-y_{2}}-c_{2}\right), \\
= & \left(\frac{\theta_{1}^{y_{1}}}{y_{1} !} e^{-\theta_{1}}\right)\left(\frac{\beta_{1}}{\theta} y_{1}-1\right) \beta_{1}^{-1}\left\{\frac{1-e^{-\theta_{1}}}{\left(\frac{\beta_{1}}{\theta_{1}} y_{1}-1\right) e^{-\theta_{1}}}\right\}^{\delta_{0}\left(y_{1}\right)}+ \\
& \left(e^{-y_{1}}-c_{1}\right) E_{\theta_{2}}\left(e^{-Y_{2}}-c_{2}\right) .
\end{aligned}
$$

Since $E_{\theta_{2}}\left(e^{-Y_{2}}-c_{2}\right)=0$, we are sure of the answer.

By symmetry, we have

$$
P\left(Y_{2}=y_{2}\right)=\left(\frac{\theta_{2}^{y_{2}}}{y_{2} !} e^{-\theta_{2}}\right)\left(\frac{\beta_{2}}{\theta} y_{2}-1\right) \beta_{2}^{-1}\left\{\frac{1-e^{-\theta_{2}}}{\left(\frac{\beta_{2}}{\theta_{2}} y_{2}-1\right) e^{-\theta_{2}}}\right\}^{\delta_{0}\left(y_{2}\right)} .
$$

(ii) $\operatorname{cov}\left(Y_{1}, Y_{2}\right)=E_{\theta_{1}, \theta_{2}}\left(Y_{1} Y_{2}\right)-E_{\theta_{1}}\left(Y_{1}\right) E_{\theta_{2}}\left(Y_{2}\right)$.

We have

$$
\begin{aligned}
E_{\theta_{1}, \theta_{2}}\left(Y_{1} Y_{2}\right)= & \sum_{y_{1}} \sum_{y_{2}} y_{1} y_{2} f_{B E P, 1}\left(y_{1}, y_{2} ; \theta_{1}, \theta_{2}, \alpha\right), \\
= & \sum_{y_{1}} \sum_{y_{2}} y_{1} y_{2} \prod_{j=1}^{2}\left[\left(\frac{\theta_{j}^{y_{j}}}{y_{j} !} e^{-\theta_{j}}\right)\left(\frac{\beta_{j}}{\theta_{j}} y_{j}-1\right) \beta_{j}^{-1}\left\{\frac{1-e^{-\theta_{j}}}{\left.\left(\frac{\beta_{j}}{\theta_{j}} y_{1}-1\right) e^{-\theta_{j}}\right\}}\right]+\right. \\
& \sum_{y_{1}} \sum_{y_{2}} y_{1} y_{2} \prod_{j=1}^{2}\left[\left(\frac{\theta_{j}^{y_{j}}}{y_{j} !} e^{-\theta_{j}}\right)\left(\frac{\beta_{j}}{\theta_{j}} y_{j}-1\right) \beta_{j}^{-1}\left\{\frac{1-e^{-\theta_{j}}}{\left.\left(\frac{\beta_{j}}{\theta_{j}} y_{j}-1\right) e^{-\theta_{j}}\right\}}\right\}^{\delta_{0}\left(y_{j}\right)}\right] \times \\
& \left(e^{-y_{1}}-c_{1}\right)\left(e^{\left.-y_{2}-c_{2}\right),}\right] \\
= & E_{\theta_{1}}\left(Y_{1}\right) E_{\theta_{2}}\left(Y_{2}\right)+\alpha E_{\theta_{1}}\left[Y_{1}\left(e^{-Y_{1}}-c_{1}\right)\right] E_{\theta_{2}}\left[Y_{2}\left(e^{-Y_{2}}-c_{2}\right)\right], \\
= & E_{\theta_{1}}\left(Y_{1}\right) E_{\theta_{2}}\left(Y_{2}\right)+\alpha\left[E_{\theta_{1}}\left(Y_{1} e^{-Y_{1}}\right)-E_{\theta_{1}}\left(Y_{1}\right) E_{\theta_{1}}\left(e^{-Y_{1}}\right)\right] \times \\
& {\left[E_{\theta_{2}}\left(Y_{2} e^{-Y_{2}}\right)-E_{\theta_{2}}\left(Y_{2}\right) E_{\theta_{2}}\left(e^{-Y_{2}}\right)\right] . }
\end{aligned}
$$


And

$$
\begin{aligned}
\operatorname{cov}\left(Y_{1}, Y_{2}\right)=\alpha & {\left[E_{\theta_{1}}\left(Y_{1} e^{-Y_{1}}\right)-E_{\theta_{1}}\left(Y_{1}\right) E_{\theta_{1}}\left(e^{-Y_{1}}\right)\right] \times } \\
& {\left[E_{\theta_{2}}\left(Y_{2} e^{-Y_{2}}\right)-E_{\theta_{2}}\left(Y_{2}\right) E_{\theta_{2}}\left(e^{-Y_{2}}\right)\right] . }
\end{aligned}
$$

Ultimately, we have $\operatorname{cov}\left(Y_{1}, Y_{2}\right)=\alpha \operatorname{cov}\left(Y_{1}, e^{-Y_{1}}\right) \operatorname{cov}\left(Y_{2}, e^{-Y_{2}}\right)$. We are sure of the answer.

Proposition 5. Under conditions (10) and (11) we have

$$
\begin{gathered}
f_{B E P, 1}\left(y_{1}, y_{2} ; \theta_{1}, \theta_{2}, \beta_{1}, \beta_{2}, \alpha\right)=\prod_{j=1}^{2}\left[\left(\frac{\beta_{j}}{\theta_{j}} y_{j}-1\right) \beta_{j}^{-1}\left\{\frac{1-e^{-\theta_{j}}}{\left(\frac{\beta_{j}}{\theta_{j}} y_{j}-1\right) e^{-\theta_{j}}}\right\}^{\delta_{0}\left(y_{j}\right)}\right] \times \\
g\left(y_{1}, y_{2} ; \theta_{1}, \theta_{2}, \alpha\right) \times f_{B P}\left(y_{1}, y_{2}, \theta_{1}, \theta_{2}\right) .
\end{gathered}
$$

Expression (22) confirms that the bivariate Poisson extended distribution is a member of the family of bivariate Poisson distributions (see [1]).

Proof. Indeed, we have

$$
\begin{aligned}
f_{B E P, 1}\left(y_{1}, y_{2} ; \theta_{1}, \theta_{2}, \beta_{1}, \beta_{2}, \alpha\right)= & \left(\frac{\theta_{1}^{y_{1}}}{y_{1} !} e^{-\theta_{1}}\right)\left(\frac{\theta_{2}^{y_{2}}}{y_{2} !} e^{-\theta_{2}}\right) \times \\
& \left(\prod_{j=1}^{2}\left[\left(\frac{\beta_{j}}{\theta_{j}} y_{j}-1\right) \beta_{j}^{-1}\left\{\frac{1-e^{-\theta_{j}}}{\left(\frac{\beta_{j}}{\theta_{j}} y_{j}-1\right) e^{-\theta_{j}}}\right\}^{\delta_{0}\left(y_{j}\right)}\right]\right) \times \\
& g\left(y_{1}, y_{2} ; \theta_{1}, \theta_{2}, \alpha\right),
\end{aligned}
$$

And under conditions (10) and (11) we are assured of the result.

\subsection{Estimation of parameters $\theta_{1}, \theta_{2}, \beta_{1}, \beta_{2}, \alpha$}

The parameters $\theta_{1}, \theta_{2}, \beta_{1}, \beta_{2}$ and $\alpha$ will be estimated by the maximum likelihood method. Let us consider an n-sample $\left(y_{1,1}, y_{2,1}\right),\left(y_{1,2}, y_{2,2}\right), \ldots,\left(y_{1, n}, y_{2, n}\right)$ of the couple of random variables $\left(Y_{1}, Y_{2}\right)$ of density $f_{B E P, 1}\left(y_{1}, y_{2} ; \theta_{1}, \theta_{2}, \beta_{1}, \beta_{2}, \alpha\right)$. The log-likelihood function $L\left(\left(y_{1}, y_{2}\right), \theta_{1}, \theta_{2}, \beta_{1}, \beta_{2}, \alpha\right)$ is given by 


$$
L\left(\left(y_{1}, y_{2}\right), \theta_{1}, \theta_{2}, \beta_{1}, \beta_{2}, \alpha\right)=\sum_{i=1}^{n} \ln f_{B E P, 1}\left(y_{1, j}, y_{2, j} ; \theta_{1}, \theta_{2}, \beta_{1}, \beta_{2}, \alpha\right) .
$$

The following system of normal equations

$$
\begin{aligned}
\frac{\partial}{\partial \theta_{j}} L\left(\left(y_{1}, y_{2}\right), \theta_{1}, \theta_{2}, \beta_{1}, \beta_{2}, \alpha\right) & =0 \\
\frac{\partial}{\partial \beta_{j}} L\left(\left(y_{1}, y_{2}\right), \theta_{1}, \theta_{2}, \beta_{1}, \beta_{2}, \beta, \alpha\right) & =0 \\
\frac{\partial}{\partial \alpha} L\left(\left(y_{1}, y_{2}\right), \theta_{1}, \theta_{2}, \beta_{1}, \beta_{2}, \alpha\right) & =0
\end{aligned}
$$

is used to calculate the estimators $\widehat{\theta}_{j}, \widehat{\beta}_{j},(j=1,2)$ and $\widehat{\alpha}$ using the package maxLik for the statistical environment $\mathrm{R}$ (see $[6]$ ).

\section{Student's t test to test $\alpha=0$}

To ensure the independance of variables $Y_{1}$ and $Y_{2}$, we must perform a statistical test that allow discriminate between the following hypotheses [9]

- Null hypothesis $H_{0}: \alpha=0$, vs

- alternative hypothesis $H_{1}: \alpha \neq 0$.

Let $\widehat{\alpha}=\widehat{\alpha}_{n}$ the maximum likelihood estimator of $\alpha$. The variable

$$
U=\sqrt{n} \frac{\widehat{\alpha}_{n}-\alpha}{\sqrt{I^{-1}\left(\alpha ; \theta_{1}, \theta_{2}, \beta_{1}, \beta_{2}\right)}}
$$

follows, when $n$ is large, the normal distribution $\mathcal{N}(0,1)$, with $I\left(\alpha ; \theta_{1}, \theta_{2}, \beta_{1}, \beta_{2}\right)$ the amount of information provided by the pair $\left(Y_{1}, Y_{2}\right)$ at parameter $\alpha$.

The result is as follows.

\section{Proposition 6.}

(i)

$$
I\left(\alpha ; \theta_{1}, \theta_{2}, \beta_{1}, \beta_{2}\right)=E_{\theta_{1}, \theta_{2}}\left[\frac{\left(e^{-Y_{1}}-c_{1}\right)^{2}\left(e^{-Y_{2}}-c_{2}\right)^{2}}{\left[1+\alpha\left(e^{-Y_{1}}-c_{1}\right)\left(e^{-Y_{2}}-c_{2}\right)\right]^{2}}\right] .
$$

(ii) And under the null hypothesis

$$
I\left(0 ; \theta_{1}, \theta_{2}, \beta_{1}, \beta_{2}\right)=\operatorname{var}\left(e^{-Y_{1}}\right) \operatorname{var}\left(e^{-Y_{2}}\right) .
$$

The estimator of $I(0 ; \theta 1, \theta 2, \beta 1, \beta 2)$ which we denote $\widehat{I}(0)$ will be calculated following two approaches: 


\section{First approach: the substitution method}

Knowing the estimators $\widehat{\theta}_{1 n}, \widehat{\theta}_{2 n}, \widehat{\beta}_{1 n}$ and $\widehat{\beta}_{2 n}$ we can estimate $I\left(0 ; \theta_{1}, \theta_{2}, \beta_{1}, \beta_{2}\right)$ by $\widehat{I}(0)=$ $I\left(0 ; \widehat{\theta}_{1 n}, \widehat{\theta}_{2 n}, \widehat{\beta}_{1 n}, \widehat{\beta}_{2 n}\right)$.

\section{Second approach: basic statistics}

Let $\bar{y}_{j n}=\frac{1}{n} \sum_{i=1}^{n} e^{-y_{j i}}$ and $s_{j n}^{\prime 2}=\frac{1}{n-1} \sum_{i=1}^{n}\left(e^{-y_{j i}}-\bar{y}_{j n}\right)^{2} \quad(j=1,2)$ the empirical mean and the empirical unbiased variance of the sample $\left(e^{-Y_{j 1}}, \ldots, e^{-Y_{j i}}, \ldots, e^{-Y_{j n}}\right) \quad(j=1,2)$ of size $n$ of the variable $e^{-Y_{j}} \quad(j=1,2)$. Since the variances $\operatorname{var}\left(e^{-Y_{1}}\right)$ and $\operatorname{var}\left(e^{-Y_{2}}\right)$ can be estimated by the respective empirical unbiased variances $s_{1 n}^{\prime 2}$ and $s_{2 n}^{\prime 2}$, then we can estimate $I\left(0 ; \theta_{1}, \theta_{2}, \beta_{1}, \beta_{2}\right)$ by $\widehat{I}(0)=s_{1 n}^{\prime 2} \times s_{2 n}^{\prime 2}$.

\section{Test statistics:}

The test statistic

$$
T=\sqrt{n} \frac{\widehat{\alpha}_{n}}{\sqrt{\widehat{I}^{-1}(0)}},
$$

follows under the null hypothesis when $n$ is large, the Student's law of degree of freedom $n$.

Decision: let $x=P(>|T|)$ the p-value. Given a first order risk $\alpha=5 \%$, if $x<\alpha$ then $H_{0}$ is rejected, otherwise it is accepted.

Proof. [Proof of the Proposition 6] We have

$$
\frac{\partial}{\partial \alpha} \ln f_{B E P, 1}=\frac{\left(e^{-Y_{1}}-c_{1}\right)\left(e^{-Y_{2}}-c_{2}\right)}{1+\alpha\left(e^{-Y_{1}}-c_{1}\right)\left(e^{-Y_{2}}-c_{2}\right)},
$$

and

Therefore

$$
\frac{\partial^{2}}{\partial \alpha^{2}} \ln f_{B E P, 1}=-\frac{\left(e^{-Y_{1}}-c_{1}\right)^{2}\left(e^{-Y_{2}}-c_{2}\right)^{2}}{\left[1+\alpha\left(e^{-Y_{1}}-c_{1}\right)\left(e^{-Y_{2}}-c_{2}\right)\right]^{2}} .
$$

$$
I\left(\alpha ; \theta_{1}, \theta_{2}, \beta_{1}, \beta_{2}\right)=E_{\theta_{1}, \theta_{2}}\left[\frac{\left(e^{-Y_{1}}-c_{1}\right)^{2}\left(e^{-Y_{2}}-c_{2}\right)^{2}}{\left[1+\alpha\left(e^{-Y_{1}}-c_{1}\right)\left(e^{-Y_{2}}-c_{2}\right)\right]^{2}}\right],
$$

and under the null hypothesis the variables $Y_{1}$ and $Y_{2}$ are independent. This leads to

$$
I\left(0 ; \theta_{1}, \theta_{2}, \beta_{1}, \beta_{2}\right)=\operatorname{var}\left(e^{-Y_{1}}\right) \operatorname{var}\left(e^{-Y_{2}}\right) \text {. }
$$

And we are sure of the answer. Expressions (6) and (7) allow us to calculate the variances of the variables $e^{-Y_{1}}$ and $e^{-Y_{2}}$. 


\section{Simulation study}

\subsection{Simulation and basic statistics}

In this section, we realize a simulation study. On this, we consider two random variables $Y_{1}$ and $Y_{2}$ following the extended Poisson distribution of respective parameters $\left(\theta_{1}, \beta_{1}\right)$ and $\left(\theta_{2}, \beta_{2}\right)$. The table 1 contains the simulations of the variables $Y_{1}$ and $Y_{2}$ and the table 2 the basic statistics. We have the presumption that according to the Fisher indices of table 2 , the variables are overdispersed. We have simulated samples of size $n=150$.

Table 1: Simulated data, $\theta_{1}=1, \beta_{1}=2$ for $Y_{1}$ and $\theta_{2}=3, \beta_{2}=5$ for $Y_{2}$

\begin{tabular}{cccccccccccc}
\hline Count & 0 & 1 & 2 & 3 & 4 & 5 & 6 & 7 & 8 & 9 & 10 \\
\cline { 1 - 9 } & 42 & 23 & 48 & 24 & 12 & 0 & 1 & & & & \\
$N_{Y_{1}}$ & 25 & 3 & 22 & 23 & 29 & 24 & 10 & 5 & 5 & 3 & 1
\end{tabular}

Table 2: Basic statistics

\begin{tabular}{cccc}
\hline Variable & Mean & Variance & Fisher index \\
\hline$Y_{1}$ & 1.6333 & 1.7371 & 1.0635 \\
\hline$Y_{2}$ & 3.4933 & 5.3657 & 1.5359 \\
\hline
\end{tabular}

\subsection{Estimation of model parameters and remark}

Using the package maxLik for the statistical environment $\mathrm{R}$ (see [6]), we have the output $\mathrm{R}$ in table 3 .

The parameter estimates are $\widehat{\theta}_{1}=1.14132, \widehat{\theta}_{2}=2.70274, \widehat{\beta}_{1}=2.64952, \widehat{\beta}_{2}=4.58362$ and $\widehat{\alpha}=1.45859$. The table 3 shows that $\alpha$ is different to 0 . Indeed, the corresponding $\mathrm{p}$-value is equal to 0.00096 , lower than at the usual significance level 0.05 , so we reject the hypothesis that $\alpha=0$ at the risk of significance $5 \%$. For this set of simulated data, the variables $Y_{1}$ and $Y_{2}$ are dependent.

\section{Conclusion}

We constructed the bivariate extended Poisson distribution as a generalization of the univariate extended Poisson distribution by the method of the product of its marginal laws by a factor. This method was demonstrated by Lakshminarayna et al.[7]. We have shown that this distribution belongs to the family of bivariate Poisson distributions. The Student's statistical test allows to highlight the independence between the variables $Y_{1}$ and $Y_{2}$. 
Table 3: Output R

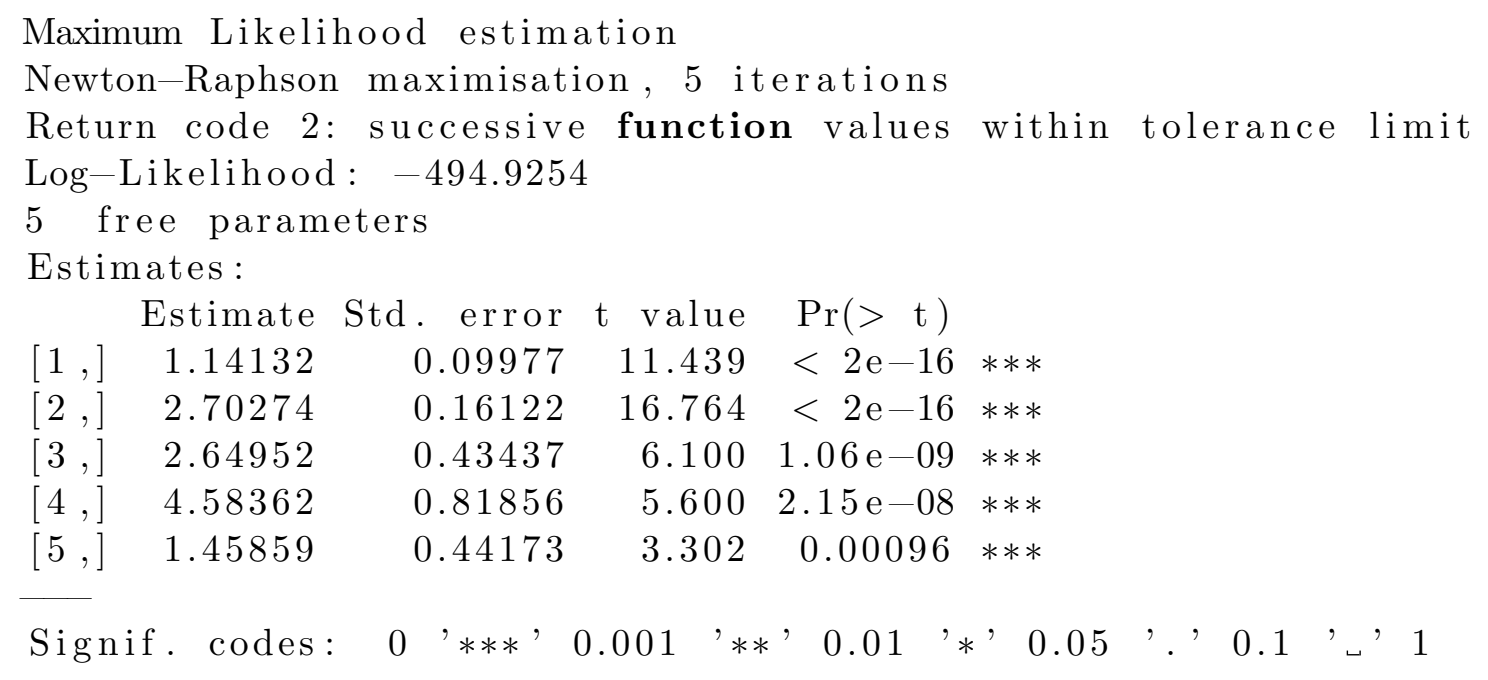

\section{References}

[1] P C Batsindila, R Bidounga, and D Mizère. The covariance structure of the biariate weighted poisson distribution and application to the aleurodicus data. Afrika Statistica, 14(2):1999-2017, 2019.

[2] P Berkhout and E A Plug. A bivariate poisson count data model using conditional probabilities. Statist Neerlandica, 58(3):349-364, 2004.

[3] R Bidounga, P C Batsindila Nganga, L Niéré, and D Mizère. A note on the (weighted) bivariate poisson distribution. European J. Pure Appl. Math., (1):192-203, 2021. Published by New York Business Global.

[4] R Bidounga, E G B Mandangui Maloumbi, R F Mizélé Kitoti, and D Mizère. The new bivariate conway-maxwel-poisson distribution obtained by crossing method. International Journal of Statistics and probability, 9(6):1-8, 2020.

[5] B Dimitrov and N Kolev. Beta transformation. beta type self-decomposition and related characterizations. Brezilian Journal of Probability ans Statistics, 14:123-140, 2000 .

[6] Arne Henningsen and Ott Toomet. maxlik: A package for maximum likelihood estimation in r. Comput Stat, (26):443-458, 2011.

[7] J Lakshminarayna, S N N Pandit, and K Srinivasa Rao. On a bivariate poisson distribution. Comm. Statist. Theory methods, 28(2):267-276, 1999. 
[8] C G Louzayadio, R O Malouata, and M Koukouatikissa Diafouka. A weighted poisson distribution for underdispersed count data. International Journal of Statistics and probability, 10(4):157-165, 2021.

[9] G Saporta. Probabilité, analyse des données et statistique. Editions Technip. Paris, 2006. 